\section{Methods to evaluate the scavenging activity of antioxidants toward reac- tive oxygen and nitrogen species}

This project objectives are: (i) to identify the quenching chemistry of biologically important reactive oxygen and nitrogen species (ROS/RNS, including free radicals); (ii) to show antioxidant action against reactive species through $\mathrm{H}$-atom and electron transfer reactions; and (iii) to evaluate the ROS/RNS scavenging activity of antioxidants with existing analytical methods while emphasizing the underlying chemical principles, advantages, and disadvantages of these methods. In addition to discussing various methodologies with respect to kinetics and thermodynamics, the project will focus on the applications and impact of existing assays on potentiating future research and innovations to evolve better methods enabling more comprehensive study of different aspects of antioxidants, and to provide a vocabulary of terms related to antioxidants and scavengers for ROS/RNS.

ROS/RNS generation is directly related to oxidative degradation in foods and biological systems. Therefore, the search for methods to determine the scavenging activity of reactive species is very important. Determination of the antioxidant/antiradical status in these systems could contribute to prevention and treatment of diseases related to aging such as cardiovascular and neurodegenerative diseases and cancer. In addition to organism defenses, the intake of dietary antioxidants and influence of real contribution of foods to antioxidant status in biological systems must be evaluated. ${ }^{1}$ The main methods comprise the scavenging activity measurement of hydroxyl radical $\left(\mathrm{HO}^{\circ}\right)$, superoxide anion radical $\left(\mathrm{O}_{2}{ }^{-{ }^{-}}\right)$, hydrogen peroxide $\left(\mathrm{H}_{2} \mathrm{O}_{2}\right)$, hypochlorous acid $(\mathrm{HOCl})$, peroxynitrite $\left(\mathrm{ONOO}^{-}\right)$, and peroxyl radical (ROO'). In spite of the diversity of methods, there is currently a great need to evaluate the scavenging activity of antioxidant compounds in vivo and in vitro. In addition, there are incorrect methods frequently used, such as non-selective UV measurement of $\mathrm{H}_{2} \mathrm{O}_{2}$ scavenging, producing negative errors due to incomplete reaction of peroxide with flavonoids in the absence of transition metal ion catalysts. $^{2}$ The Project Chair's research collaborators have recently published a comprehensive review on "methods of measurement and evaluation of natural antioxidant capacity/activity."3 Complementary to this technical report, this project will provide chemical information about methods clarifying reaction mechanisms, thermodynamic scavenging efficiency, and kinetic scavenging rates of antioxidants. Moreover, it will aid the identification of reactive species and quantification of scavenging extents of antioxidants through various assays, make the results comparable and more understandable, and bring a more rational basis to the evaluation of these assays. Modern nano-technological methods for the estimation of reactive species and their scavenging action will also be discussed.

\section{References}

1. B. Halliwell, J.M.C. Gutteridge, Free radicals in biology and medicine, 4th edition, Oxford University Press, Oxford, (2007).

2. M. Ozyurek, B. Bektasoglu, K. Guclu, N. Gungor, R. Apak, J. Food Compos. Anal. 23 (2010) 689-698.

3. R. Apak, S. Gorinstein, V. Boehm, K. M. Schaich, M. Ozyurek, K. Guclu, Pure Appl. Chem. 85 (2013) 957-998.

For more information, contact the Task Group Chair Resat Apak $<$ rapak@istanbul.edu.tr>

Sint www.iupac.org/project/2013-015-1-500

\section{Development and Dissemination of a Green Chemistry Curriculum for Latin American and African Universities}

The green chemistry philosophy was introduced around 20 years ago. Since then, a growing number of studies contemplating a diversity of strategies to integrate green principles into chemistry educationmainly at the higher education level-have been carried out. ${ }^{1,2}$ According to some authors, ${ }^{3}$ the efforts to incorporate green chemistry contents into Chemistry courses indicate that a problem-oriented approach substantially promotes a more effective training when a topic pertaining to the local context is also considered. Thus, the present project aims at developing, optimizing and testing the introduction of green chemistry components into modules for the chemistry degrees curricula in Latin American and African universities, taking the regional reality into account.

Modules based on the Science-Technology-Society (STS) teaching approach have already been proposed and tested in undergraduate classes. The proposed lessons begin with the presentation and discussion of documentaries with emphasis on controversial socio-scientific issues, followed by a lecture based on 